\title{
Guillain-Barré syndrome after SARS-CoV-2 vaccination in a patient with previous vaccine-associated Guillain-Barré syndrome
}

\author{
Ling Ling MD, Sean M. Bagshaw MD MSc, Pierre-Marc Villeneuve MD MSc
}

Cite as: CMAJ 2021 November 22;193:E1766-9. doi: 10.1503/cmaj.210947

A 63-year-old man presented to the emergency department with progressive, ascending weakness of the upper and lower extremities. The patient had a history of hypertension, dyslipidemia, insulin-dependent diabetes mellitus, nephrolithiasis, obesity, anxiety and Guillain-Barré syndrome (GBS) after vaccination against influenza. His medications were insulin glargine, metformin, semaglutide, gliclazide, amlodipine, valsartan, atorvastatin and potassium citrate.

In September 1999, about 3 weeks after receiving a vaccine against seasonal influenza, the patient developed acute facial paralysis, ascending upper and lower extremity weakness and paresthesias, progressing to flaccid paralysis of all 4 extremities, accompanied by generalized areflexia. No abnormalities were found on a computed tomography scan of his head at the time. Lumbar puncture showed elevated levels of cerebrospinal fluid (CSF) protein. Nerve conduction studies were not done, but GBS was diagnosed based on clinical presentation and investigations. The patient required prolonged mechanical ventilation and tracheostomy and was treated with systemic corticosteroids and intravenous immune globulin. He eventually achieved nearcomplete neurologic recovery with only mild residual facial palsy. The patient had received his childhood vaccinations without adverse effects, but this had been his first vaccination against influenza and he did not have any further vaccinations until the SARS-CoV-2 pandemic.

Twelve days before presentation to the emergency department, he received his first dose of the ChAdOx1 nCoV-19 vaccine (AstraZeneca), a replication-deficient, simian adenovirus vaccine to SARS-CoV-2. He developed mild chills and fatigue after vaccination that resolved within 3 days. Eleven days later, he developed paresthesias of the lips and fingers on both hands. On the day of presentation, he developed rapidly progressive and ascending weakness in his upper and lower extremities. He had had no preceding symptoms that suggested an infection.

At presentation, physical examination showed mild weakness $(4+/ 5$, using the Medical Research Council's scale for muscle strength) of both shoulder abductors and knee flexors, though the patient's strength was otherwise preserved. He had areflexia, bilateral loss of pinprick and vibration sensation up to the mid-shin,

\section{Key points}

- Guillain-Barré syndrome (GBS) has very rarely been described after vaccination, but a causal relationship has not been established.

- GBS should be considered in the differential diagnosis of a patient who presents with symmetric, ascending muscle weakness, with sensory deficits, after vaccination against influenza and SARS-CoV-2.

- More cases of GBS have been reported after SARS-CoV-2 infection than after vaccination; the benefits of SARS-CoV-2 vaccination outweigh the potential adverse effects for nearly all patients.

- The risks and benefits of SARS-CoV-2 vaccination should be carefully discussed with patients who have a history of GBS after previous vaccinations.

with no sensory level, and loss of proprioception to the ankle joint. His gait was abnormally wide. After admission, his weakness progressed to $1 / 5$ strength in his ankle dorsiflexors, $1 / 5$ strength in the extensor hallucus longus, 3-4/5 strength in his knee extensors, $1-2 / 5$ hip flexors' strength, and 4/5 strength in his shoulder abductors, elbow flexors, extensors and wrist extensors, bilaterally.

Electrophysiologic studies showed sensorimotor peripheral neuropathy with substantial demyelinating features and superimposed axonal features, consistent with a diagnosis of GBS (Figure 1, Table 1). Analysis of the patient's CSF showed albinocytologic dissociation (i.e., elevated protein without leukocytosis), with a normal white blood cell count of 1 (normal $\leq 5) \times 10^{6} / \mathrm{L}$, elevated protein of 0.6 (normal $0.15-0.45$ ) $\mathrm{g} / \mathrm{L}$, elevated glucose at 6.6 (normal 2.2-4.4) $\mathrm{mmol} / \mathrm{L}$ (serum glucose $11.5 \mathrm{mmol} / \mathrm{L}$ ) and lactate of 2.8 (normal <2.8) $\mathrm{mmol} / \mathrm{L}$. We could not find any evidence of an infectious, metabolic or structural cause for his symptoms. Specifically, CSF bacterial cultures were negative and nucleic acid testing for enterovirus, parechovirus, varicellazoster virus and herpes simplex virus type 1 and 2 were negative. Serum investigations were negative for hepatitis $B$, hepatitis $C$, HIV and syphilis infections. Blood cultures were sterile. Thyroid and adrenal function and electrolytes were normal. A computed 


\section{A Motor}
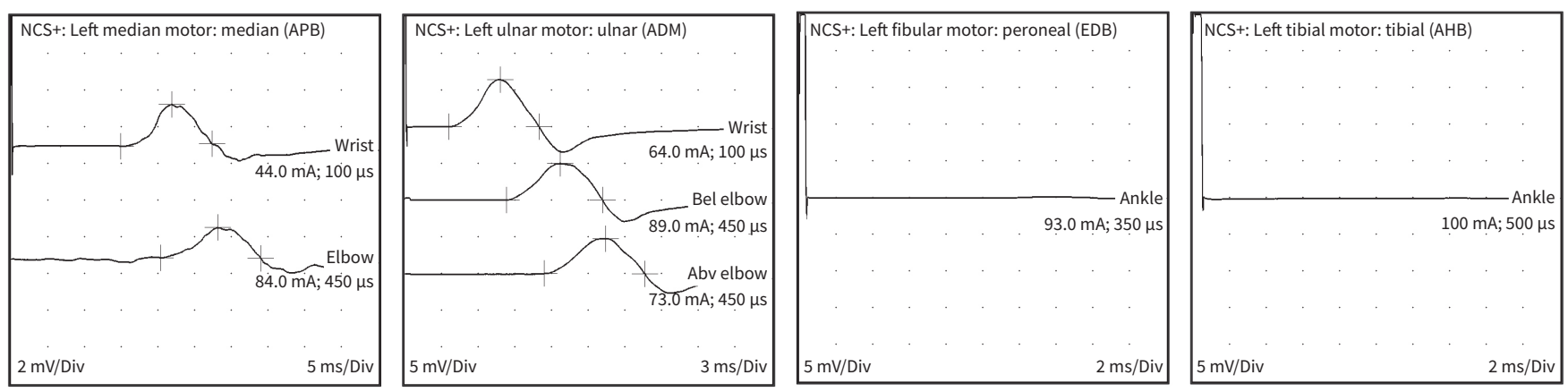

B Sensory
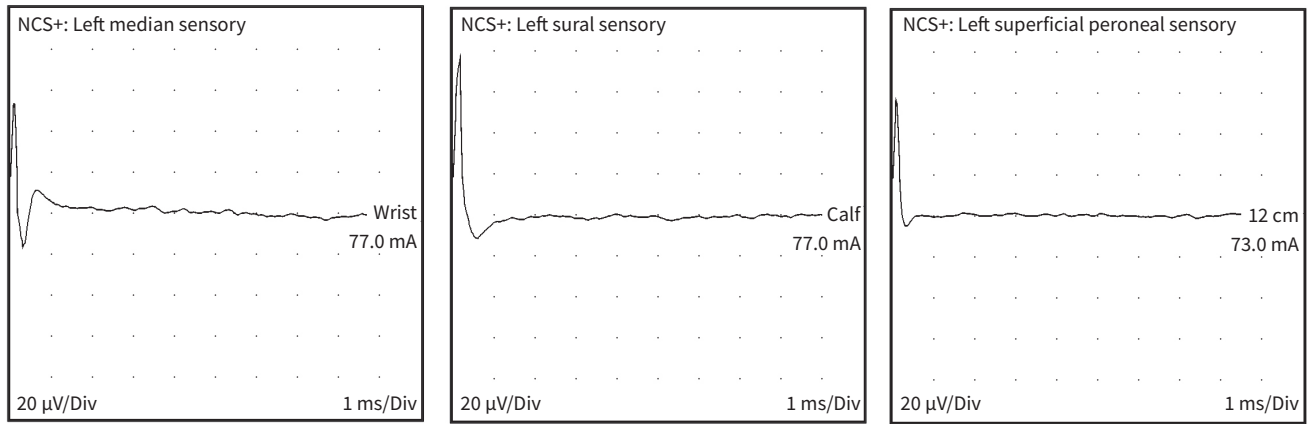

Figure 1: Waveforms from (A) motor and (B) sensory nerve conduction studies of a 63-year-old man with Guillain-Barré syndrome (GBS). These nerve conduction studies are compatible with GBS, with both axonal and demyelinating features, as shown by decreased velocity and decreased amplitude. The motor deficit was most pronounced in the left fibular (peroneal) and tibial nerves, as shown by the complete lack of conduction. A severe sensory deficit was also present in the sensory nerves studied. Note: $\mathrm{Abv}=$ above, $\mathrm{AHB}=$ abductor hallucis brevis, $\mathrm{ADM}=\mathrm{abductor}$ digiti minimi, $\mathrm{APB}=\mathrm{abductor}$ pollicis brevis, $\mathrm{Bel}=$ below, $\mathrm{EDB}=$ extensor digitorum brevis, $\mathrm{mA}=$ milliamps, $\mathrm{ms} / \mathrm{Div}=$ millisecond per division, $\mathrm{mV} /$ Div $=\mathrm{millivolt} / \mathrm{division}$, $\mathrm{NCS}+=$ nerve conduction study, $\mu \mathrm{s}=$ microseconds.

tomography scan of the patient's head did not show any acute abnormality, though we observed background small vessel ischemic disease. Magnetic resonance imaging of the cervical spine showed a normal cervical spinal cord. Antihuman neuronal ganglioside serology is not routinely performed in our centre in typical cases of GBS, and was not done.

We initially treated the patient with intravenous immune globulin. However, as his motor weakness and vital capacity deteriorated, we subsequently performed therapeutic plasma exchange. In addition to usual supportive management, the patient required 3 days of noninvasive mechanical ventilation, but never received invasive mechanical ventilation.

Three months after presentation, our patient's motor function had improved substantially. He had persistent mild weakness (4/5) in his hands and proximal and distal lower extremities, areflexia, abnormal light touch, and pinprick and vibration sensation.

The Clinical Immunology and Allergy consult service indicated that no in vitro test was available to definitively identify any trigger for an episode of GBS. The temporal relation between vaccination and onset of symptoms 12 days later was expected, as GBS is not an immunoglobulin (Ig) E-mediated process like anaphylaxis.

We suggested that the patient avoid future doses of the ChAdOx1 nCoV-19 vaccine. Little evidence exists to guide a recommendation for this patient regarding the safety of SARS-CoV-2 vaccines that do not use a viral vector.

\section{Discussion}

Guillain-Barré syndrome is an immunologically mediated, inflammatory, demyelinating polyneuropathy. We describe a 63-year-old man who developed GBS about 12 days after receiving his first dose of the ChAdOx1 nCoV-19 vaccine. He had had a previous episode of GBS that presented 3 weeks after receiving seasonal influenza vaccination in 1999.

Guillain-Barré syndrome is thought to be caused by antibody- (IgG) and immune cell-mediated (T-cell, macrophage) destruction of the myelin and axonal membranes of peripheral neurons. An event such as infection, use of medication (e.g., immune checkpoint inhibitor), vaccination or surgery usually precedes the syndrome. Although there is no consensus definition of vaccine-associated GBS, vaccination is considered relevant if received up to 4 weeks before onset of symptoms. ${ }^{1}$ However, whether vaccination is truly a trigger for GBS remains uncertain. A meta-analysis of 39 studies found an increased relative risk of 1.41 (95\% confidence interval [Cl] 1.20-1.66) of GBS after influenza vaccination, ${ }^{2}$ but this was not found in a subsequent large, singlecountry, retrospective study of GBS onset within 180 days after vaccination. ${ }^{3}$

Infection with SARS-CoV-2 has been reported to precede GBS; 73 cases were reported in 1 study. ${ }^{4}$ We found several case reports of GBS occurring after SARS-CoV-2 vaccination, relating to both the ChAdOx1 nCoV-19 (AstraZeneca) and BNT 162b2 (Pfizer) vaccines..$^{5-8}$ 
Table 1: Nerve conduction studies from a 63-year-old man with Guillain-Barré syndrome*

\begin{tabular}{|c|c|c|c|c|c|c|}
\hline Sites & Latency, ms & Amplitude, mV & $\begin{array}{c}\text { Negative area, } \\
\text { ms*mV }^{\star} \mathbf{m V}\end{array}$ & $\begin{array}{l}\text { Minimum F-wave } \\
\text { latency, ms }\end{array}$ & Distance, cm & $\mathrm{CV}, \mathrm{m} / \mathrm{s}$ \\
\hline \multicolumn{7}{|l|}{ Motor sites } \\
\hline Wrist & 14.9 & 2.3 & 13.7 & NR & 7 & \\
\hline Elbow & 20.4 & 1.67 & 11.5 & & 23 & 42 \\
\hline Wrist & 3.6 & 6.4 & 23.5 & 37.6 & 6 & \\
\hline Below elbow & 8.3 & 5.0 & 21.8 & & 27 & 57 \\
\hline Above elbow & 11.4 & 4.8 & 21.5 & & 11 & 35 \\
\hline \multicolumn{7}{|l|}{ Left radial (EIP) } \\
\hline Forearm & 2.9 & 5.4 & 26.9 & & 8 & \\
\hline \multicolumn{7}{|l|}{ Left fibular (EDB) } \\
\hline Ankle & NR & NR & NR & & 9 & \\
\hline \multicolumn{7}{|c|}{ Left fibular (tibial anterior) } \\
\hline Fibular head & 4.5 & 3.8 & 24.4 & & - & \\
\hline Popliteal fossa & 7.5 & 3.8 & - & & 10 & 33 \\
\hline \multicolumn{7}{|l|}{ Left tibial (AHB) } \\
\hline Ankle & NR & NR & NR & & - & \\
\hline \multicolumn{7}{|l|}{ Left facial (nasalis) } \\
\hline \multicolumn{7}{|l|}{ Left ulnar } \\
\hline Wrist-digit V & NR & NR & NR & NR & 12 & NR \\
\hline \multicolumn{7}{|l|}{ Left sural } \\
\hline Calf-latency mall & NR & NR & NR & NR & 14 & NR \\
\hline \multicolumn{7}{|c|}{ Left superficial peroneal } \\
\hline $12 \mathrm{~cm}$ AMM & NR & NR & NR & NR & 12 & NR \\
\hline
\end{tabular}

Note: $\mathrm{ADM}=$ abductor digiti minimi, $\mathrm{AHB}=$ abductor hallucis brevis, $\mathrm{AMM}=$ anterior medial malleolar, $\mathrm{APB}=$ abductor pollicis brevis, $\mathrm{CV}=$ conduction velocity, $\mathrm{EDB}=$ extensor digitorum brevis, EIP = extensor indicis proprius, NR = no response.

*Latency is the time it takes for the impulse to generate a response. In demyelination, this is prolonged, as shown in the latency times for the wrist (normal $<2.2 \mathrm{~ms}$ ) and elbow (normal $<4.4 \mathrm{~ms}$ ) of the left median APB, and at the wrist of the left ulnar ADM (normal $<2.2 \mathrm{~ms}$ ). F-wave is a measure of motor conduction time of the entire nerve. In demyelination, this is prolonged, as shown in the left ulnar ADM.

Previous case reports have estimated the risk of recurrent GBS at $1 \%-6 \%,{ }^{9}$ compared with an estimated incidence of 1.11 cases per 100000 person-years ${ }^{1}$ in the general population. Therefore, the likelihood of coincidentally developing GBS shortly after vaccination is higher in someone who has a previous history of GBS than someone who does not. In a survey, 126 patients with previous GBS received a vaccine against influenza and none reported recurrence of symptoms. ${ }^{10}$ Pritchard and colleagues found that in 29 patients who had a vaccine within 6 weeks before an initial diagnosis of GBS, $2(6.9 \%, 95 \% \mathrm{Cl} 0.85 \%-22.8 \%)$ had recurrence of generally mild symptoms after a subsequent unrelated vaccine. ${ }^{11}$ These results were based on patient survey and confirmation of recurrent GBS was not documented. 
Little evidence exists to guide the evaluation of risk for development of recurrent vaccine-related GBS. The Centers for Disease Control and Prevention published guidance in 2010 based on data from the Vaccine Adverse Event Reporting System and the United Kingdom's General Practice Research Database, recommending that, in general, patients who developed GBS within 6 weeks of receiving the influenza vaccine should not be vaccinated for influenza again if they are not at high risk of severe influenza complications. ${ }^{12}$ In 2012, the American Academy of Allergy, Asthma and Immunology issued a similar recommendation. ${ }^{13}$ The Public Health Agency of Canada advises that future influenza vaccination is generally contraindicated if GBS occurred within 6 weeks of influenza vaccination, although the risks of GBS related to the vaccine should be weighed against the risk of GBS from influenza infection. ${ }^{14}$ It is not clear whether this recommendation is generalizable to other vaccines (e.g., SARS-CoV-2 vaccines) or in patients with recurrent GBS associated with vaccination. In these rare scenarios, clinicians should have a careful risk-benefit discussion with their patient, describing the relative lack of evidence and considering individual patient circumstances.

No convincing evidence has shown that SARS-CoV-2 vaccination causes GBS, and this case report does not imply such causation. In Canada, active surveillance for neurologic events, including GBS, is conducted by the immunization monitoring program, IMPACT, funded by the Public Health Agency of Canada. ${ }^{15}$, Immunization is a vital intervention to reduce transmission and illness from SARS-CoV-2, and absolute contraindications to vaccination are rare.

\section{References}

1. Shahrizaila N, Lehmann HC, Kuwabara S. Guillain-Barré syndrome. Lancet 2021;397:1214-28.

2. Martin Arias LH, Sanz R, Sáinz M, et al. Guillain-Barré syndrome and influenza vaccines: a meta-analysis. Vaccine 2015;33:3773-8.

3. Chen Y, Zhang J, Chu X, et al. Vaccines and the risk of Guillain-Barré syndrome. Eur J Epidemiol 2020;35:363-70.

4. Abu-Rumeileh S, Abdelhak A, Foschi M, et al. Guillain-Barré syndrome spectrum associated with COVID-19: an up-to-date systematic review of 73 cases. $J$ Neurol 2021;268:1133-70.

5. Patel SU, Khurram R, Lakhani A, et al. Guillain-Barré syndrome following the first dose of the chimpanzee adenovirus-vectored COVID-19 vaccine, ChAdOx1. BMJ Case Rep 2021;14:e242956. doi: 10.1136/bcr-2021-242956.

6. Hasan T, Khan M, Khan F, et al. Case of Guillain-Barré syndrome following COVID19 vaccine. BMJ Case Rep 2021;14:e243629. doi: 10.1136/bcr-2021-243629.

7. Trimboli M, Zoleo P, Arabia G, et al. Guillain-Barré syndrome following BNT162b2 COVID-19 vaccine. Neurol Sci 2021 Aug 4;1-2. doi: 10.1007/s10072-021-05523-5. [Epub ahead of print].

8. McKean N, Chircop C. Guillain-Barré syndrome after COVID-19 vaccination. BMJ Case Rep 2021; Jul 30;14(7):e244125. doi: 10.1136/bcr-2021-244125.

9. Basta I, Bozovic I, Berisavac I, et al. Recurrent Guillain-Barré syndrome - case series. Neurol India 2019;67:1536-8.

10. Kuitwaard K, Bos-Eyssen ME, Blomkwist-Markens PH, et al. Recurrences, vaccinations and long-term symptoms in GBS and CIDP. J Peripher Nerv Syst 2009;14:310-5.

11. Pritchard J, Mukherjee R, Hughes RAC. Risk of relapse of Guillain-Barré syndrome or chronic inflammatory demyelinating polyradiculoneuropathy following immunisation. J Neurol Neurosurg Psychiatry 2002;73:348-9.
12. Fiore AE, Uyeki TM, Broder K, et al. Prevention and control of influenza with vaccines: recommendations of the Advisory Committee on Immunization Practices (ACIP), 2010. MMWR Recomm Rep 2010;59:1-62.

13. Kelso JM, Greenhawt MJ, Li JT, et al. Adverse reactions to vaccines practice parameter 2012 update. J Allergy Clin Immunol 2012;130:25-43.

14. Contraindications, precautions, and concerns: Canadian Immunization Guide. Ottawa: Government of Canada; 2020. Available: https://www.canada.ca/en/public -health/services/publications/healthy-living/canadian-immunization-guide-part -2-vaccine-safety/page-3-contraindications-precautions-concerns.html\#p2c2t1 (accessed 2021 Apr. 3).

15. Bettinger JA, Halperin SA, Vaudry W, et al.; Canadian IMPACT members. The Canadian Immunization Monitoring Program, ACTive (IMPACT): Active surveillance for vaccine adverse events and vaccine-preventable diseases. Can Commun Dis Rep 2014;40(Suppl 3):41-4. doi: 10.14745/ccdr.v40is3a06. Available: https://www. canada.ca/en/public-health/services/reports-publications/canada-communicable -disease-report-ccdr/monthly-issue/2014-40/ccdr-volume-40-s-3-december-4-2014 /ccdr-volume-40-s-3-december-4-2014-3.html (accessed 2021 Apr. 10).

Competing interests: Sean Bagshaw reports grants, consulting fees and honoraria from Baxter, outside the submitted work. He participates on the data safety monitoring board of the I-SPY-COVID-19 trial and the advisory board of BioPorto. No other competing interests were declared.

This article has been peer reviewed.

The authors have obtained patient consent.

Affiliations: Department of Medicine, Faculty of Medicine and Dentistry (Ling, Bagshaw), University of Alberta and Alberta Health Services; Department of Critical Care and Medicine, Division of Internal Medicine (Villeneuve), University of Alberta and Grey Nuns Hospitals, Edmonton, Alta.

Contributors: Ling Ling and Pierre-Marc Villeneuve reviewed the patient's chart and imaging, and drafted the manuscript. Sean Bagshaw reviewed the content, and analyzed and interpreted the data. All of the authors revised the manuscript critically for important intellectual content, approved the final version to be published and agreed to be accountable for all aspects of the work.

Content licence: This is an Open Access article distributed in accordance with the terms of the Creative Commons Attribution (CC BY-NCND 4.0) licence, which permits use, distribution and reproduction in any medium, provided that the original publication is properly cited, the use is noncommercial (i.e., research or educational use), and no modifications or adaptations are made. See: https://creativecommons.org/ licenses/by-nc-nd/4.0/

Correspondence to: Pierre-Marc Villeneuve, pierremarc.villeneuve@albertahealthservices.ca

The section Cases presents brief case reports that convey clear, practical lessons. Preference is given to common presentations of important rare conditions, and important unusual presentations of common problems. Articles start with a case presentation (500 words maximum), and a discussion of the underlying condition follows (1000 words maximum). Visual elements (e.g., tables of the differential diagnosis, clinical features or diagnostic approach) are encouraged. Consent from patients for publication of their story is a necessity. See information for authors at www.cmaj.ca. 